\title{
Benchmark Problem for Calculating Oxygen Potential in High Burnup LWR Fuel using the THERMOCHIMICA Module in Moose/Bison
}

September 30, 2014

\section{Prepared by}

S. Simunovic, T.M. Besmann, S.L. Voit

Approved for public release:

distribution is unlimited.

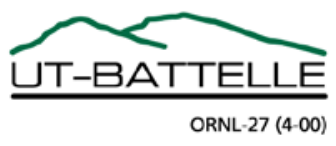




\section{DOCUMENT AVAILABILITY}

Reports produced after January 1, 1996, are generally available free via the U.S. Department of Energy (DOE) Information Bridge.

Web site http://www.osti.gov/bridge

Reports produced before January 1, 1996, may be purchased by members of the public from the following source.

National Technical Information Service

5285 Port Royal Road

Springfield, VA 22161

Telephone 703-605-6000 (1-800-553-6847)

TDD 703-487-4639

Fax 703-605-6900

E-mail info@ntis.gov

Web site http://www.ntis.gov/support/ordernowabout.htm

Reports are available to DOE employees, DOE contractors, Energy Technology Data Exchange (ETDE) representatives, and International Nuclear Information System (INIS) representatives from the following source.

Office of Scientific and Technical Information

P.O. Box 62

Oak Ridge, TN 37831

Telephone 865-576-8401

Fax 865-576-5728

E-mail reports@osti.gov

Web site http://www.osti.gov/contact.html

This report was prepared as an account of work sponsored by an agency of the United States Government. Neither the United States Government nor any agency thereof, nor any of their employees, makes any warranty, express or implied, or assumes any legal liability or responsibility for the accuracy, completeness, or usefulness of any information, apparatus, product, or process disclosed, or represents that its use would not infringe privately owned rights. Reference herein to any specific commercial product, process, or service by trade name, trademark, manufacturer, or otherwise, does not necessarily constitute or imply its endorsement, recommendation, or favoring by the United States Government or any agency thereof. The views and opinions of authors expressed herein do not necessarily state or reflect those of the United States Government or any agency thereof. 
Computer Science and Mathematics Division Materials Science and Technology Division

Fusion and Materials for Nuclear Systems Division

\title{
BENCHMARK PROBLEM FOR CALCULATING OXYGEN POTENTIAL IN HIGH BURNUP LWR FUEL USING THE THERMOCHIMICA MODULE IN MOOSE/BISON
}

\author{
S. Simunovic, T. M. Besmann, S.L .Voit
}

Date Published: September 2014

Prepared by

OAK RIDGE NATIONAL LABORATORY

Oak Ridge, Tennessee 37831-6283

managed by

UT-BATTELLE, LLC

for the

U.S. DEPARTMENT OF ENERGY

under contract DE-AC05-00OR22725 



\section{Executive Summary}

A new thermochemical model has been developed for representing irradiated fuel with fission products. Calculations of oxygen potential as a function of burnup were performed using the developed model, and when clad oxidation is considered the results well reproduce the limited available measurements. The thermochemical equilibrium solver software library THERMOCHIMICA was used for modeling the oxidation state of LWR fuel during irradiation. The local oxygen chemical potential and its gradient are considered to be the most important chemical properties of nuclear fuel because they strongly affect many diffusion controlled processes and oxidation of the inner surface of the fuel cladding. New computer subroutines and functions were implemented in MOOSE/BISON software for calculation of oxygen partial pressure, oxygen to metal ratio in the fuel fluorite phase, and the oxygen chemical potential. Experimental measurements from the open literature were used to validate the implemented models and illustrate functionality of the developed thermodynamics module. This process involved conducting neutronics, isotopic depletion transmutation and decay of the fuel using the SCALE system. The isotopic predictions using SCALE and thermochemical prediction using THERMOCHIMICA models resulted in a good agreement with the available experimental measurements. The developed THERMOCHIMICA modules constitute a unique capability for modeling composition and spatial variation of compounds and phases in the nuclear fuel during irradiation, and provide the framework for more accurate modeling of diffusion controlled processes in the multicomponent systems. The THERMOCHIMICA modules were also coupled with the existing burnup kernel and isotopic models in BISON. Simulations of the existing benchmark problems in Bison were conducted to validate the model implementation.

Notice: This manuscript has been authored by UT-Battelle, LLC, under Contract No. DE-AC0500OR22725 with the U.S. Department of Energy. The United States Government retains and the publisher, by accepting the article for publication, acknowledges that the United States Government retains a non-exclusive, paid-up, irrevocable, world-wide license to publish or reproduce the published form of this manuscript, or allow others to do so, for United States Government purposes. 


\section{Introduction}

The evolving chemical composition of nuclear fuel during irradiation and the corresponding thermochemical properties strongly influence the fuel's behavior in normal operating conditions and during transients [1]. The chemical state influences physical properties and response of the fuel including thermal conductivity, heat capacity, swelling, creep, fission product volatility, etc. [2]. As LWR $\mathrm{UO}_{2}$ fuels are burned, they generate up to sixty transuranic and fission product elements resulting in a complex chemical system [3]. To represent that system a thermodynamic database is under development that can be used with equilibrium solvers to determine the local chemical state, including vapor pressures and oxygen potential. To date the database contains assessed representations of U-Pu-O, U-La-O, U-Gd-O, U-Ce-O as plutonium and the lanthanoids are high concentration fission products that readily dissolve in $\mathrm{UO}_{2}$ and have a strong influence on behavior. Thus these together can accurately predict behavior within the combined systems in fuel. Other important fission products are also included in the database, along with secondary phases, only a subset of which has been assessed. Model approximations in the form of ideal solutions in the fluorite phase are being used for the remainder of the systems, and these can provide reasonable results pending completion of the remaining assessments of important fission products. Noble metal phases are included based on a reported assessment.

THERMOCHIMICA [4] is a software library developed for calculation of thermochemical equilibrium at a prescribed composition, temperature and pressure. The equilibrium solver determines a unique combination of phases and their compositions at thermochemical equilibrium. The computational algorithms and software were developed at the Royal Military College of Canada and at the Oak Ridge National Laboratory and have been released in the public domain. THERMOCHIMICA routines were developed for incorporation of equilibrium thermo-chemical calculations into other simulation codes, with emphasis on nuclear fuel problems. Considerable research efforts have been undertaken to achieve high computational performance, primarily through advanced algorithm development, such as improved estimation techniques and domain tailored nonlinear solvers $[5,6]$.

Thermochemical equilibrium calculations are only one of the tools for estimation of the nuclear fuel's chemical state and behavior. During irradiation, the fission products are continuously generated and change due to transmutation and decay, the oxygen chemical potential changes with the burnup and production of fission products, and most importantly, temperature and chemical potential gradients affect the formation of various phases and species transport and spatial distribution [3]. Therefore, a thermochemistry equilibrium solver needs to be a part of a larger multiphysics simulation system in order to achieve its full potential.

THERMOCHIMICA was incorporated into the MOOSE (Multiphysics Object Oriented Simulation Environment) [7] code that was developed by the Idaho National Laboratory. MOOSE is a computational framework for development of simulation tools for solving coupled, nonlinear partial differential equations (PDEs). BISON [8] is a finite element method (FEM) code built within MOOSE for simulation of coupled multi-physics phenomena in nuclear fuel elements and nuclear reactor engineering. The physical phenomena at the continuum scale are modeled by conservation laws expressed by PDEs and the coupling of the physics models is then formulated and modeled by systems of coupled PDEs. A detailed description of the implementation of THERMOCHIMICA can be found in a technical report [9].

The task reported in this document involves implementation of the modeling capabilities for calculation of oxygen chemical potential and oxygen to metal ratio in the fluorite phase of nuclear 
fuel. The developed thermodynamic models and their BISON implementation were verified with experimental measurements of composition and oxygen potential of the high burnup fuel [10]. The verification generally follows the procedure established in a recent journal publication by Piro et al. [1] and thus the details of the procedure will not be explained in full detail here. We describe only the main steps of the simulation workflow and compare the simulation results to the experimental data. In order to verify the developed thermodynamic model and its implementation into BISON for high-burnup LWR fuel, we have performed full neutronics, isotopic depletion, transmutation and decay calculations in the SCALE [11] system for the fuel composition and power history used in the experiments [10]. The experimental data for model verification includes oxidation and local fission product concentration measurements on the same sample. The isotopic composition calculated by the SCALE system was used in thermochemical equilibrium calculations for the high burnup fuel. The developed thermodynamic models and subroutines were implemented into BISON which was used for the verification calculations. The isotopic predictions using SCALE and thermochemical prediction using values and models in THERMOCHIMICA resulted in good agreement with the available experimental measurements. The developed subroutines were also coupled with the existing burnup and isotope models in BISON [12] and their performance was demonstrated using the program's assessment and example problems.

\section{THERMOCHIMICA Implementation for Nuclear Fuel}

THERMOCHIMICA software consists of a library of material thermodynamic properties, a select set of thermodynamic models, and a numerical solver to minimize global Gibbs energy while respecting the Gibbs Phase Rule and conservation of mass. THERMOCHIMICA utilizes input parameters and a specific model framework to predict a unique combination of species and phases that are stable at thermodynamic equilibrium and then outputs material properties and boundary conditions. Specifically, the software uses input parameters such as temperature, pressure, and species mass together with a thermodynamic database such as that described above, to calculate species mole fraction, chemical potentials, thermochemical activities, moles of phases present, enthalpy, heat capacity, and Gibbs energy.

THERMOCHIMICA solves for thermodynamic equilibrium and does not take chemical kinetic information into consideration. It is implicit to the method that the chemical reactions are controlled by thermodynamics and not kinetics. In reality, chemical equilibrium is not achieved instantaneously. However, chemical kinetics are not very significant at sufficiently high temperatures, long time periods, and when atoms of the various chemical elements are randomly mixed in the system. In nuclear fuels under normal operating conditions, chemical equilibrium is achieved quickly due to the high temperatures, and the chemical reactions occur over long time periods between refueling. Also, due to the nature of fission, the atoms of the various elements representing the transuranics and fission products are randomly mixed in irradiated nuclear fuel. This implicit assumption of local thermodynamic equilibrium must be taken into account in model development and in the solution procedures in the code.

\section{Thermochemical Database for LWR Nuclear Fuel}

A global LWR nuclear fuel database has been under development at ORNL. It is currently based on the original work of Gueneau, et al. [13] who developed a thermochemical model for the fluorite structure urania phase with solutes, and in their work plutonium was considered as a solute. The model utilizes the compound energy formalism (CEF), which in this case allows for consideration of cation solutes and oxygen vacancies and interstitials. 
The urania-plutonia CEF model has been expanded to include all the relevant rare earth fission products as well as yttrium, zirconium, molybdenum, and tellurium. Given the fact that fission product concentrations will be relatively dilute, a good assumption was that interactions would occur only between uranium and the respective other cations in the structure. Thus thermochemical models of urania with the fission products integrated into a single CEF model for the fluorite phase would be sufficiently accurate. To date, assessed models for urania with plutonium, lanthanum, cerium, gadolinium (as a burnable poison) have been included in the urania representation. The remaining cations are so far being treated as ideal solution components, which will be adequate for the demonstration of fuel behavior in the current work.

Secondary phases will from in the urania fuel, although most only at relatively high burnups [14]. Phases that form at lower burnups include the white or noble metal phase. These are alloys of Rh, $\mathrm{Ru}, \mathrm{Mo}, \mathrm{Pd}$, and Tc which can be present in the bcc, fcc, or hcp structures. Thermochemical models for these phases have been adopted from the work of Kaye, et al. [15].

A model for the perovskite phase formed from fission products and seen in high burnup oxide fuel was generated as a solid solution of $\mathrm{BaO}, \mathrm{SrO}, \mathrm{Cs}_{2} \mathrm{O}, \mathrm{ZrO}_{2}, \mathrm{MoO}_{2}, \mathrm{RbO}_{2}$, and actinide dioxides. This model remains unoptimized, but the phase does appear in calculations of fuel at high burnup and is seen as adequately representative.

Other secondary oxide phases are observed at high burnups in fuel and are represented in the database. These include $(\mathrm{Ba}, \mathrm{Si}) \mathrm{O},(\mathrm{Rb}, \mathrm{Cs})_{2} \mathrm{ZrO}_{3},(\mathrm{Sr}, \mathrm{Ba}) \mathrm{ZrO}_{3},(\mathrm{Rb}, \mathrm{Cs})_{2} \mathrm{UO}_{4},(\mathrm{Sr}, \mathrm{Ba}) \mathrm{UO}_{4}$, and(Rb,Cs) $\mathrm{MoO}_{4}$.

\section{Thermochemical Modeling of Burnup}

The elemental compositions provided from the burnup calculations performed in ORIGEN were used as input to thermochemical equilibrium calculations. The FactSage [16] software package was used for computing the equilibrium state utilizing the database described above. The equilibrium oxygen potential was determined, and for convenience defined by

\section{$R T \ln p_{O 2}$}

where $R$ is the ideal gas law constant, $T$ is temperature, and $p_{O 2}$ is the oxygen partial pressure. For these calculations the total pressure was 1 bar and temperature was $1023 \mathrm{~K}$. The conditions were selected to match those of reported oxygen potential measurements on irradiated fuel samples to allow direct comparison.

The computed oxygen potentials together with reported measurements are shown in Fig. 1. The blue curve containing relatively more positive oxygen potential values are the result of the direct calculation of oxygen potential based on the composition of fuel at the specific burnup values. The results can be seen to be generally more positive than the reported measurements of Adamson, et al. [17], Une, et al. [18], Matzke [19], and Walker, et al. [10]. The calculations, however, do not take into account the observed oxidation of the inner zirconium clad surface and therefore the resultant loss of oxygen from the fuel. In order to represent the oxidation of the clad and therefore to better fit the measured fuel oxidation potentials, the equivalent of the amount of oxygen that would be lost to $\mathrm{ZrO}_{2}$ was removed from the calculations. The surface area of clad directly adjacent to a fuel pellet was assumed to oxidize, and the depth of the oxide layer was assumed to increase from $0.7 \mu \mathrm{m}$ at $12.5 \mathrm{GWd} / \mathrm{t}$ to a steady state $6 \mu \mathrm{m}$ at $>50 \mathrm{GWd} / \mathrm{t}$ to fit the observed oxygen potential values. The internal cladding oxide layer thickness also agrees well with the typical observation of about a $6 \mu \mathrm{m}$ 
layer reported by Walker, et al. [10]. The orange, more negative oxygen potential curve in Figure 1 is seen to well represent the limited reported measurements.

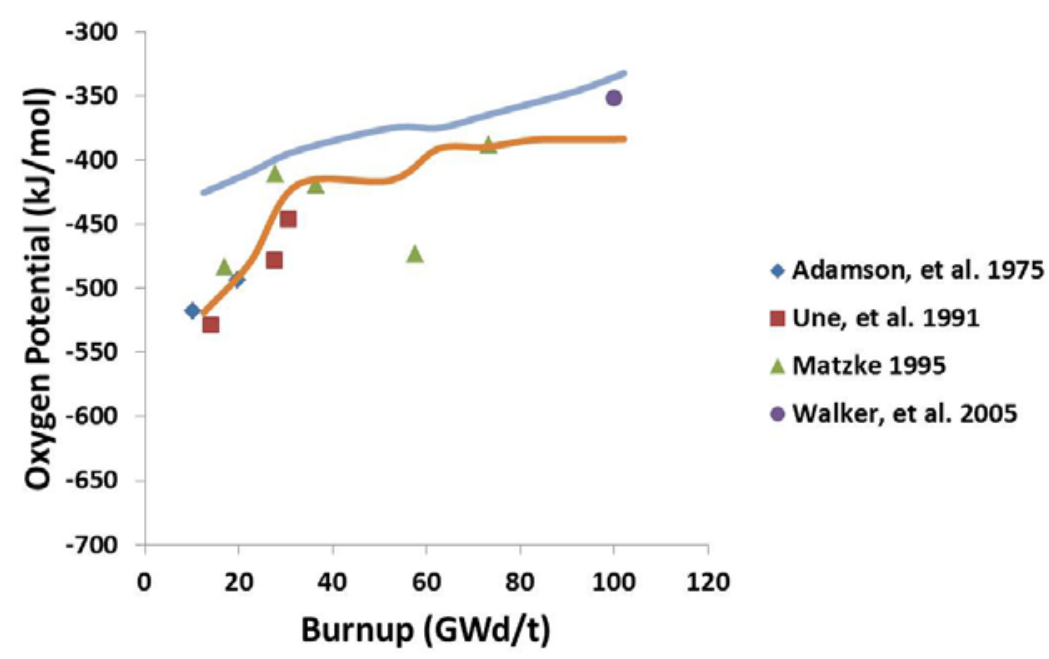

Figure 1. Computed oxygen potentials (lines) as a function of burnup for PWR fuel compared to reported measurements (points) [10, 17-19]. The blue curve represents direct equilibrium calculations from the ORIGEN results, whereas the orange curve includes oxygen loss due to clad oxidation.

\section{SCALE Simulation for High Burnup LWR Fuel}

In order to simulate isotope composition of high burnup LWR fuel, we performed a full SCALE simulation for the fuel and power profile of Walker et al. [10]. The irradiation history of the fuel is shown in Figure 2.

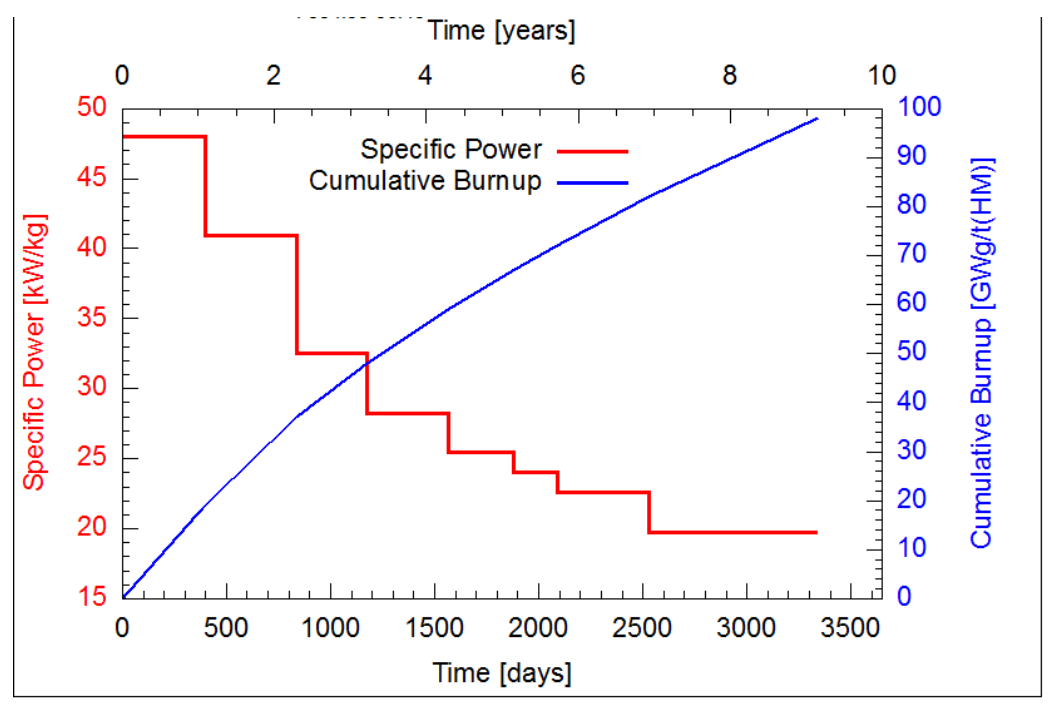

Figure 2. The irradiation history of $\mathrm{UO}_{2}$ fuel used in the current simulation.

The initial unirradiated fuel was slightly hyperstoichiometric with an enrichment of $3.5 \%$. The outer 
diameter of the fuel was $9.3 \mathrm{~mm}$ and had $95 \%$ of theoretical density. The fuel was that of a commercial Siemens 15x15 PWR fuel assembly. It was irradiated over a period of 9 power cycles to an integral burnup of $102 \mathrm{GWd} / \mathrm{t}(\mathrm{U})$. The SCALE 2D model configuration is shown in Figure 3.

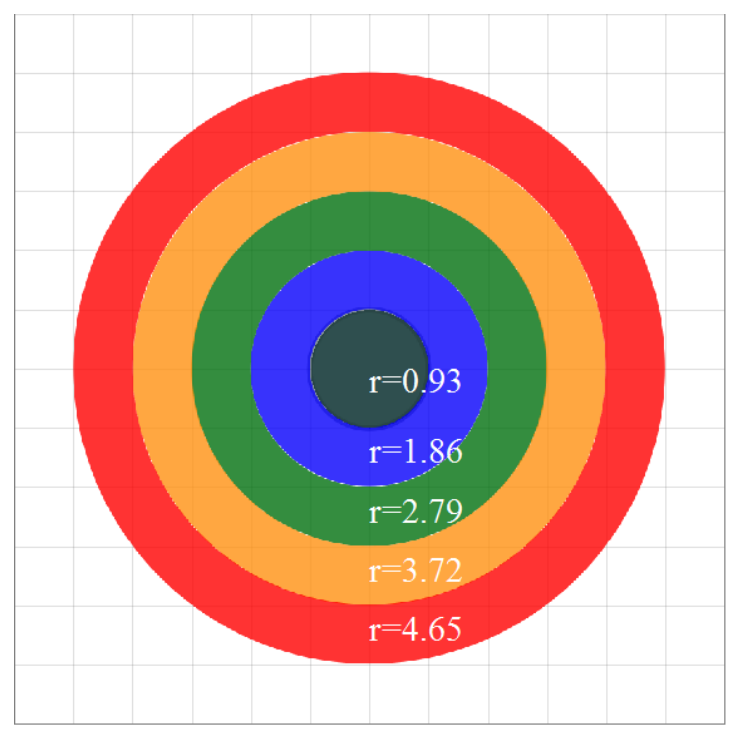

Figure 3. SCALE model geometry where $r$ is the outer radius of the annular section in mm.

In contrast with the procedure employed in Piro et al. [1], a multi zone SCALE model was used. This approach accounts for the feedback of the self-shielding of cross sections and a new transport solution during burnup. In order to provide an adequate resolution of elemental distribution in the high burnup regions, many more rings would be needed. However, for the purpose of validation of the current model, the above configuration was considered to be sufficient. A high resolution model is currently under development and will be the subject of subsequent effort. The details of the SCALE system calculations can be found in references [1] and [11]. The resulting predicted average fission product inventory in the fuel and its comparison with experimental measurements [10] is shown in Figure 4.

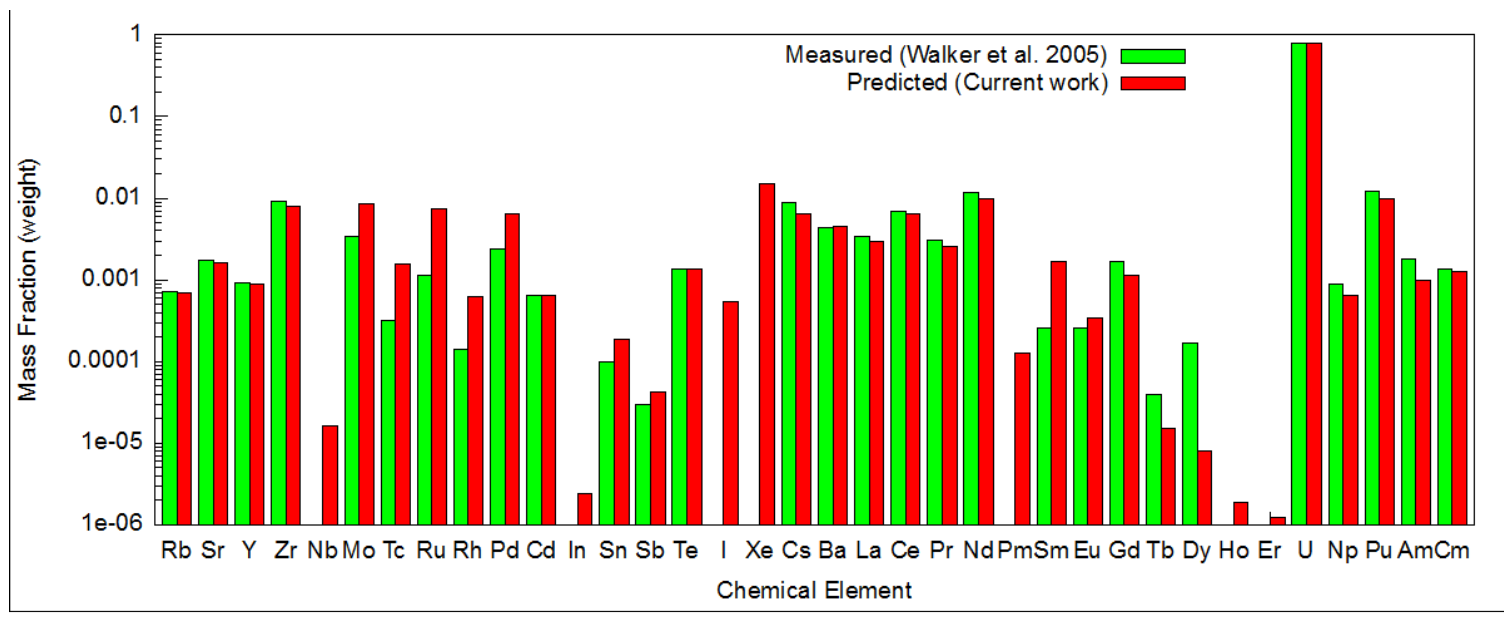

Figure 4. The predicted average chemical element inventory compared to experimental measurements [10]. 
The predicted results are in a good agreement with the experimental measurements. The predicted radial distribution of the element inventory is shown in Figure 5.

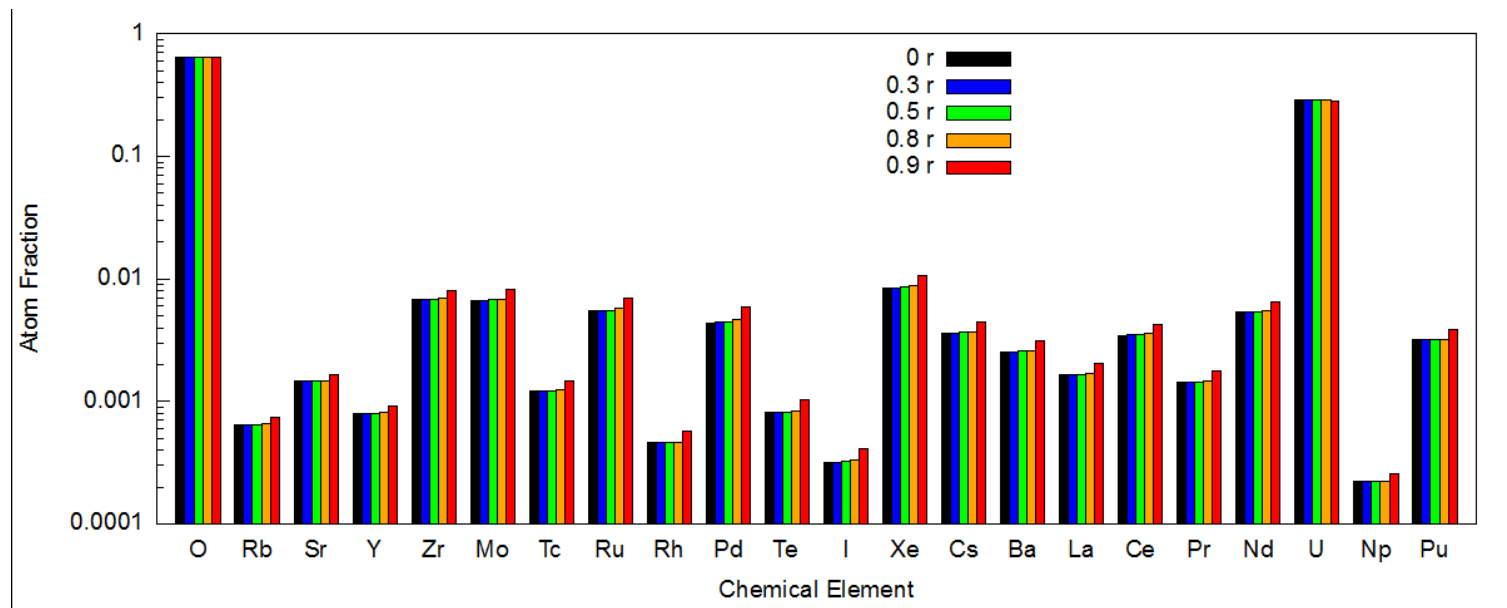

Figure 4. The predicted distribution of chemical element inventory along the radius of the pellet.

The modeling of the high burnup region, i.e., the rim region, can be enhanced by using a larger number of ring elements in the SCALE simulation.

\section{Modeling of the Oxidation State of the LWR Nuclear Fuel Material}

The chemical element composition for different radial locations of nuclear fuel was used in thermodynamic calculations of the oxidation state of the fuel using the implemented subroutines in BISON. The results are summarized in Figures 5 and 6 . Figure 5 shows the oxygen potential as a function of temperature for different locations in the fuel compared to experimental measurements. The measurements for the location $r / r_{0}=0.1$ are presented for completeness only. They should not be used in the overall evaluation as it was reported by Walker et al. [10] that the measurements in this region were not reproducible and had large errors. 


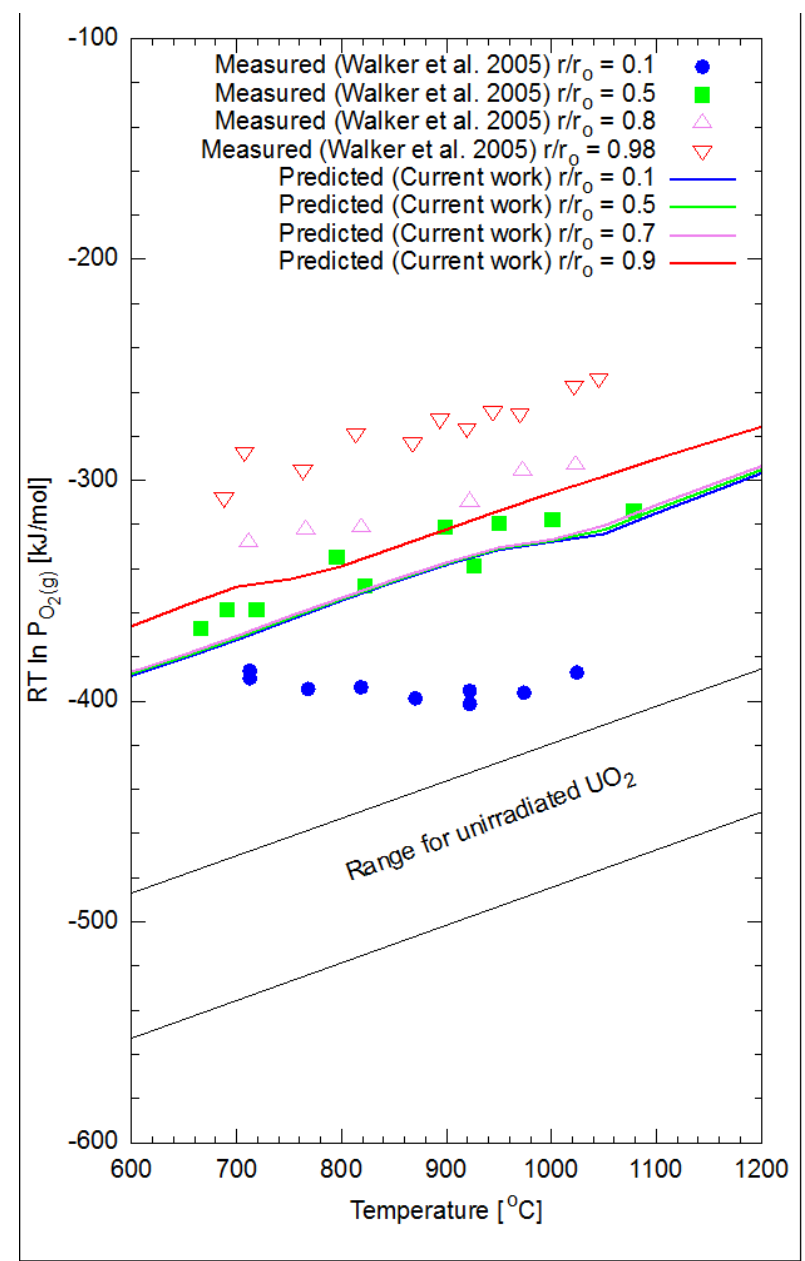

Figure 5. The predicted oxygen potential compared to experimental measurements of Walker et al. [10].

Note that the locations of the predicted values do not coincide exactly to the experimental measurements due to the specific discretization used in SCALE. Nevertheless, the predicted results are close to those of the experiments, especially considering that the experiments had an estimated error of $10 \%$ [1]. The range for unirradiated $\mathrm{UO}_{2}$ is shown for comparison. The predicted variation of the oxygen potential along the radius of the pellet is shown in Figure 6. 


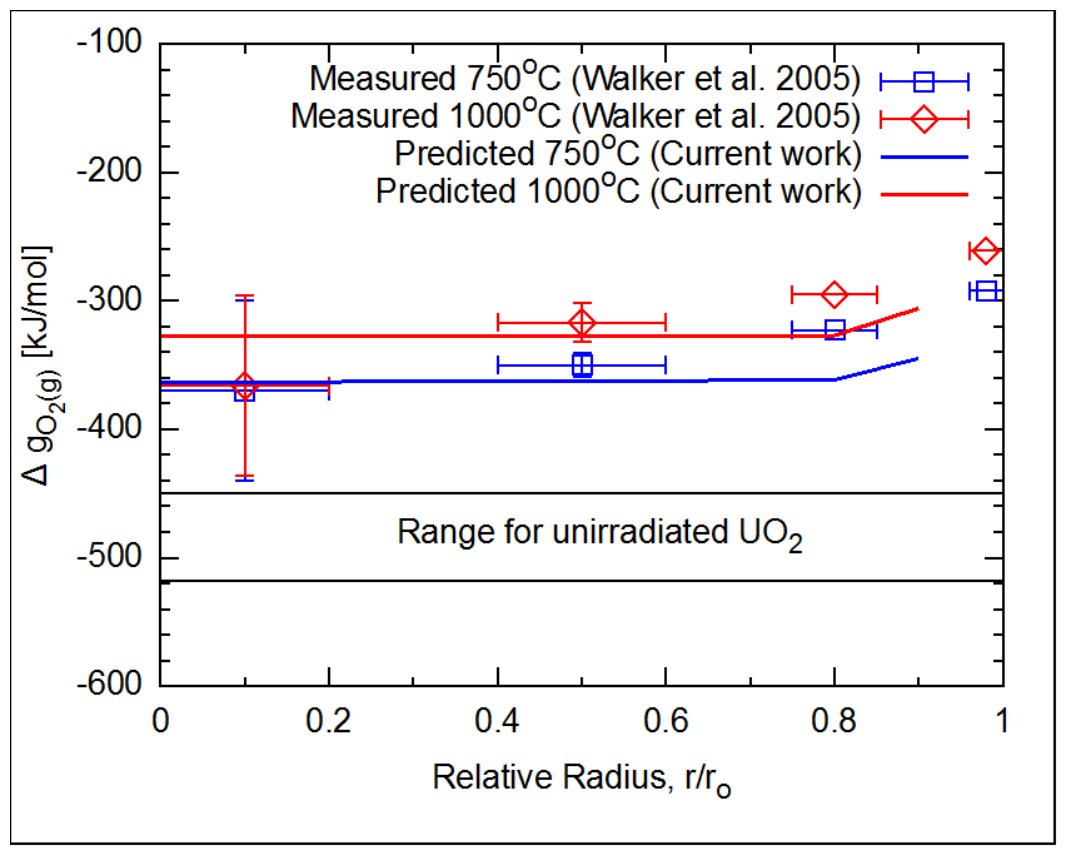

Figure 6. The predicted oxygen potential distribution along the radius of the pellet compared to experimental measurements of Walker et al. [10].

Taking into consideration that the SCALE computation only had 5 zones along the radius of the pellet, and that the burnup and thus fission products concentration increases rapidly in the submillimeter, rim zone in the pellet periphery, the predicted results show good agreement with the experiments.

\section{THERMOCHIMICA Implementation of Oxidation-Related Calculations in BISON}

New modules for oxidation-related calculations were implemented in BISON. In the MOOSE nomenclature, the implementations consist of Initial Condition module (02PPressureIc . C) and Auxiliary Kernel modules (02PPressureAux . C and OxygenThermochimicaAux . C). The Auxiliary Kernel 02PPressureAux . $C$ was developed for interfacing with the existing burnup module in BISON and uses only uranium and plutonium isotopes. An example for the Initial Condition input for 02PPressureIc . C is shown in Listing 1. 


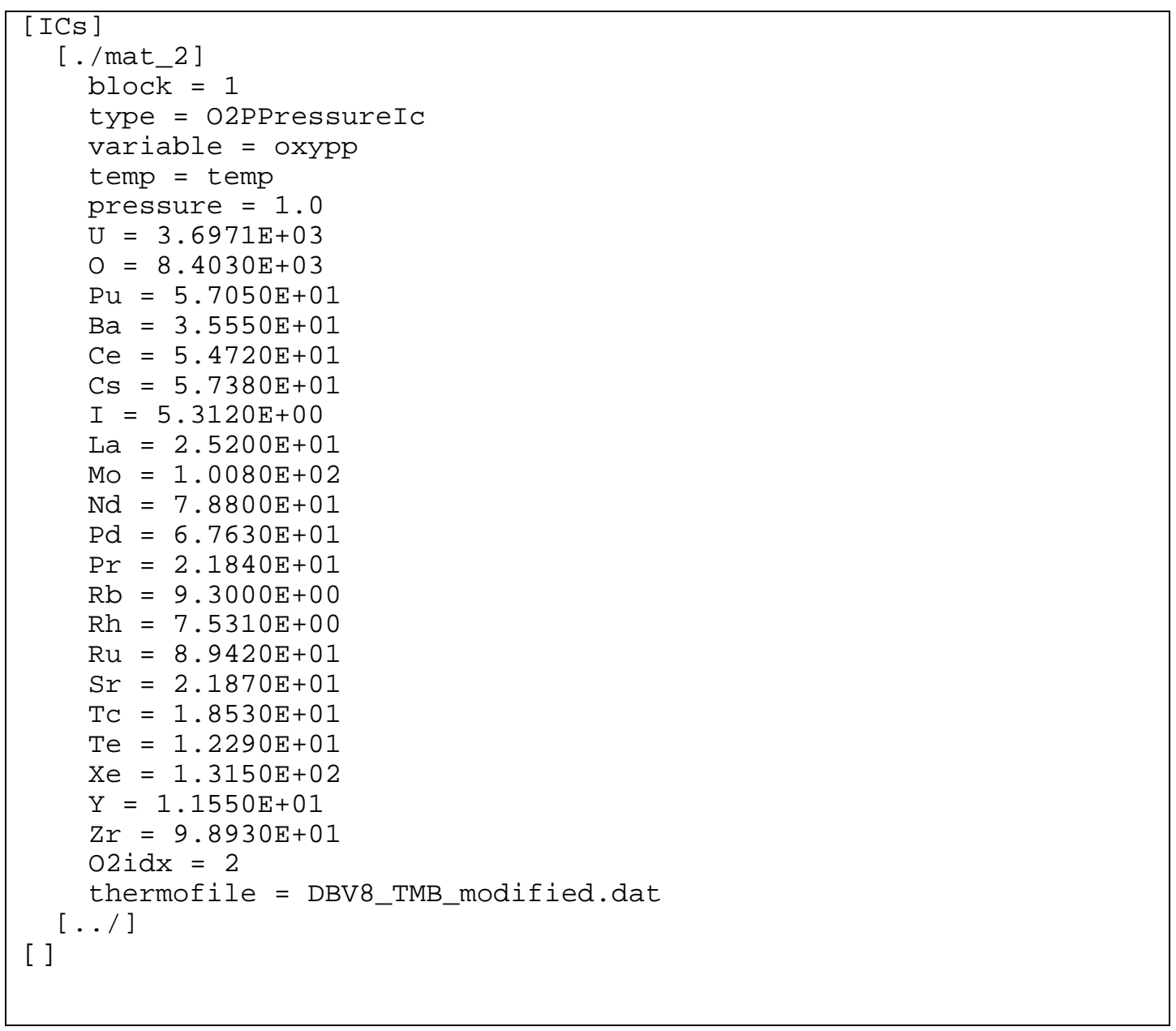

Listing 1. An example of the input syntax for the calculation of initial conditions for the oxidation state of the fuel in BISON.

The element symbols define amounts of chemical elements in the fuel. Parameter 02idx denotes the index of the $\mathrm{O}_{2}$ species in the calculation that is based on the thermodynamic model in the thermofile database. A partial listing of the initial condition implementation is given in Listing 2 . Only the main FORTRAN subroutine calls are shown in the listing. Error checking and other data transformations are omitted. 


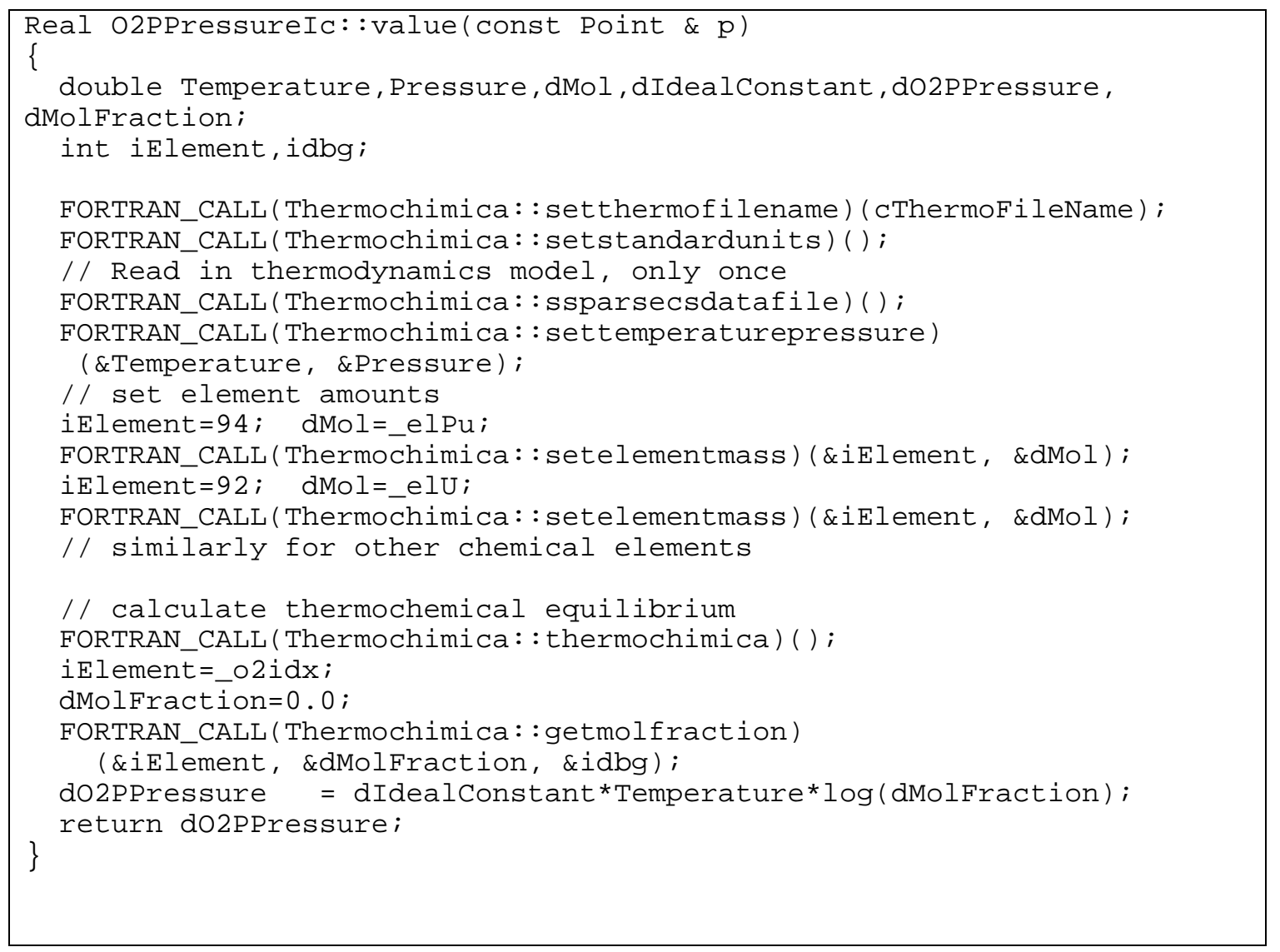

Listing 2. An example of the input syntax for the calculation of initial conditions for the oxidation state of the fuel in BISON.

Definition of the object construction and initiation in MOOSE and BISON is fairly straightforward and is not shown in the above listing. The initial condition can be coupled to the temperature field. An added benefit of the framework is that the initial condition is valid for both regular and aux kernel variables.

The main component of the implementation of the oxidation auxiliary kernel 0xygenThermochimicaAux.C is shown in Listings 3 and 4. Listing 3 shows the constructor of the kernel. It couples temperature and provides for the calculation of oxygen potential and oxygen to metal ratio in the fuel fluorite phase. 


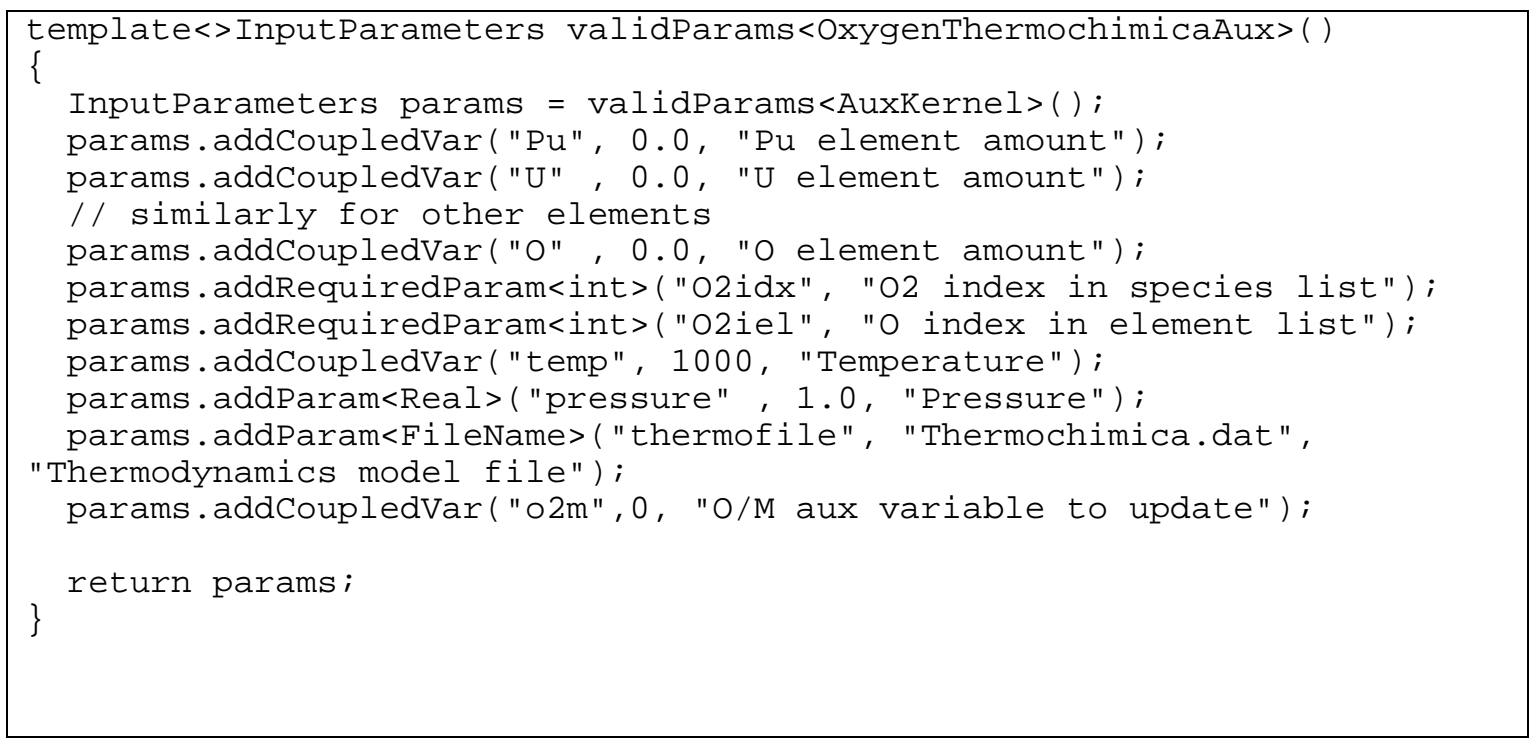

Listing 3. Constructor of OxygenThermochimicaAux auxiliary kernel.

The main auxiliary kernel evaluation steps are shown in Listing 4.

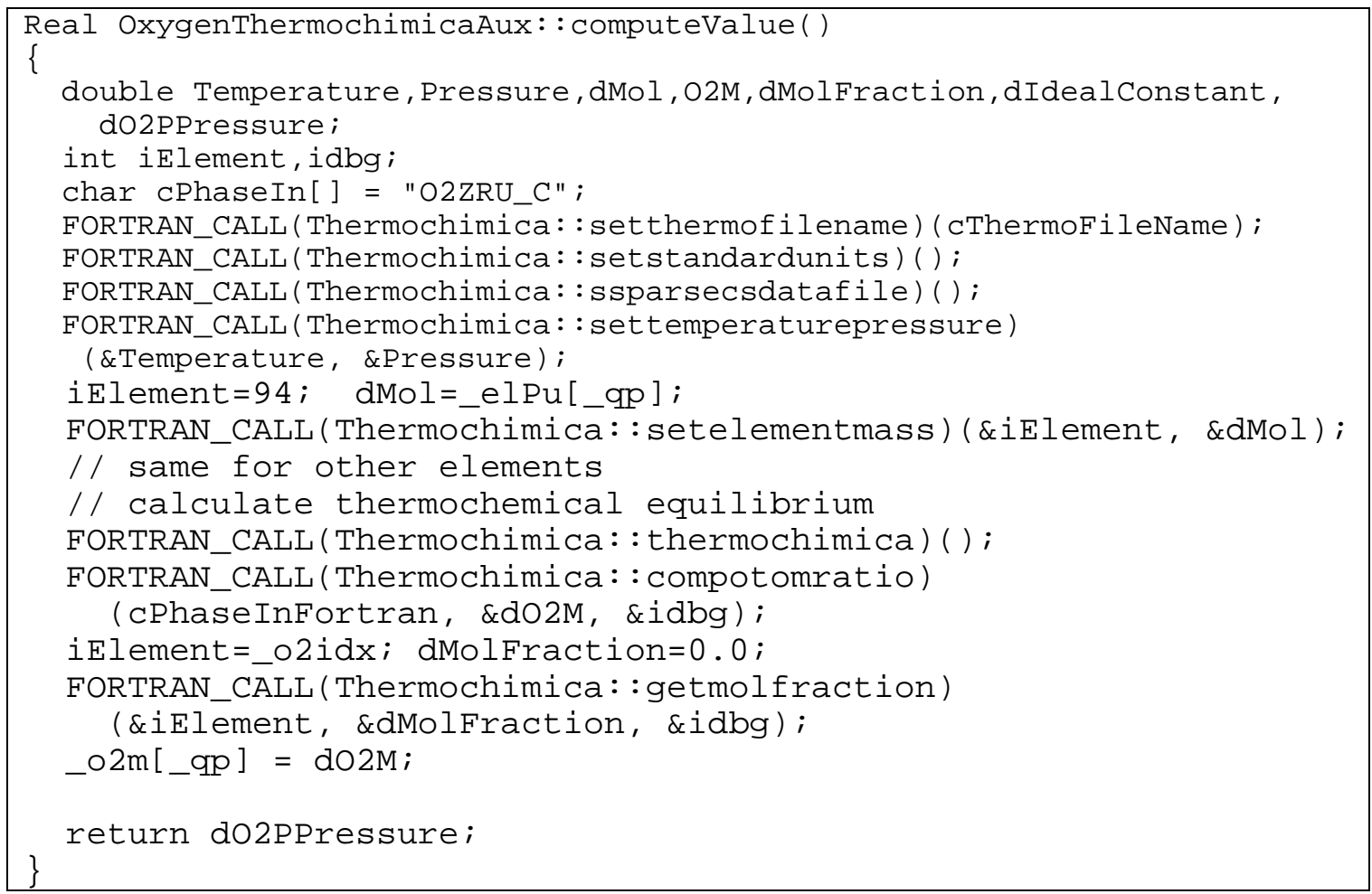

Listing 4. Aux kernel OxygenThermochimicaAux evaluation procedure.

As can be seen, the oxygen potential denoted as oxypp is the main variable. This was imposed because the experimental data was in that form. The main variable may be changed during further development as we will concentrate on the modeling of oxygen diffusion in fuel. An example of the input for the OxygenThermochimicaAux is shown in Listing 5. 


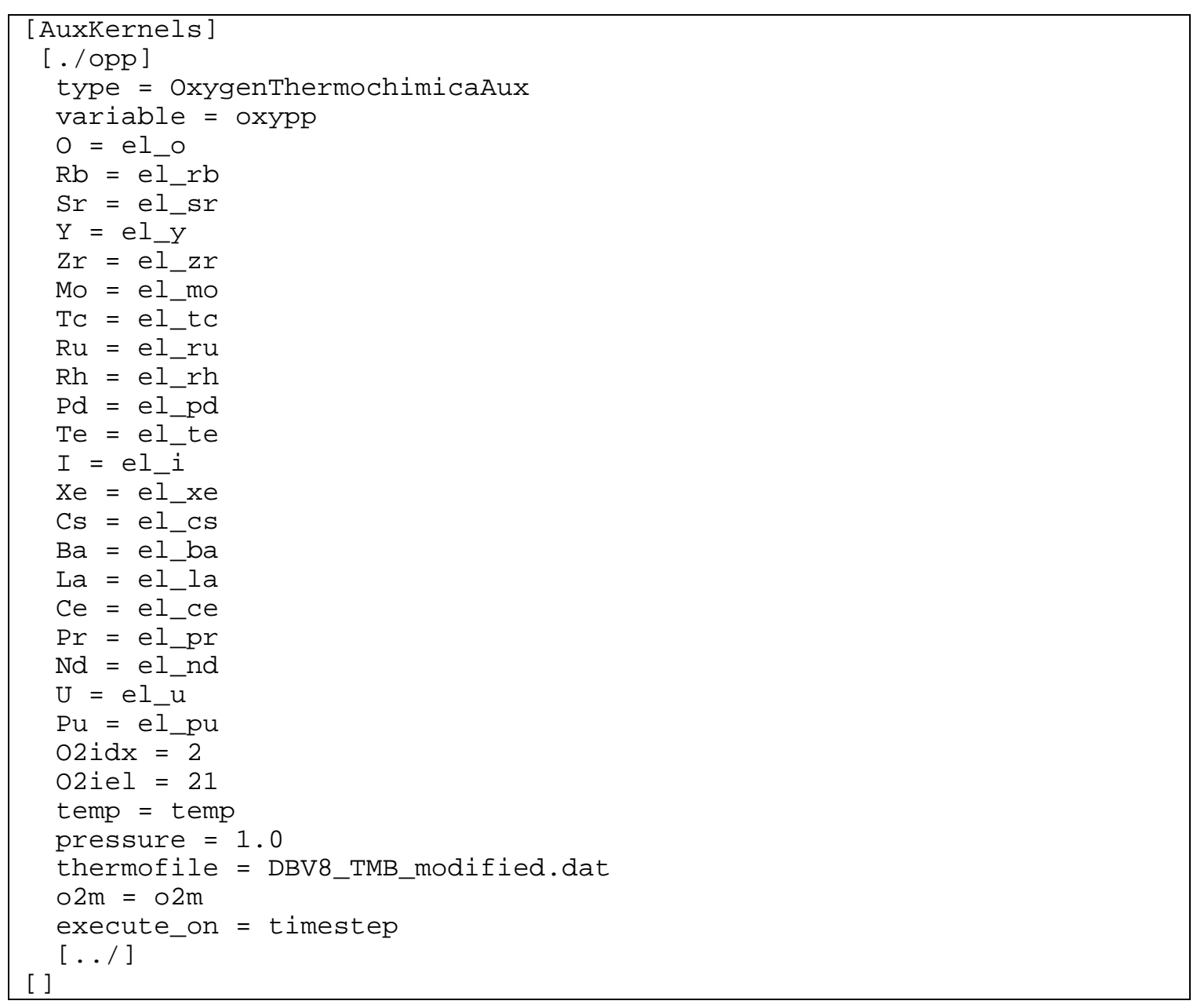

Listing 5. Example input for 0xygenThermochimicaAux auxiliary kernel.

The parameters 02idx denote index of $\mathrm{O}_{2}$ in the species list and oxygen in the element list of the thermodynamic model. The thermodynamic model file is specified by thermofile parameter. Parameter $02 \mathrm{~m}$ denote aux variable for the oxygen to metal ratio in the fluorite phase. Element concentrations are defined as separate auxiliary variables that may be provided by other BISON modules.

A separate implementation of an auxiliary kernel, OxygenPPressureAux, that couples the THERMOCHIMICA thermodynamic calculations with the existing burnup and isotope model [12] in BISON (Burnup kernel) has also been utilized. BISON's burnup model can calculate the amount of uranium and plutonium isotopes evolving during burnup, denoted as N235, N238, N239, N240, N241, and N242. The isotopes for each element are summed and used together with the initial amount of oxygen in the fuel for calculating the thermochemical equilibrium in the U-Pu-O system, and related oxidation state parameters. This is a simple system that does not take advantage of the full capabilities of the thermochemical solver, but it is used here for illustration of a potential of coupling between different physics phenomena. An example of the input block for the developed kernel OxygenPPressureAux is shown in Listing 6. 


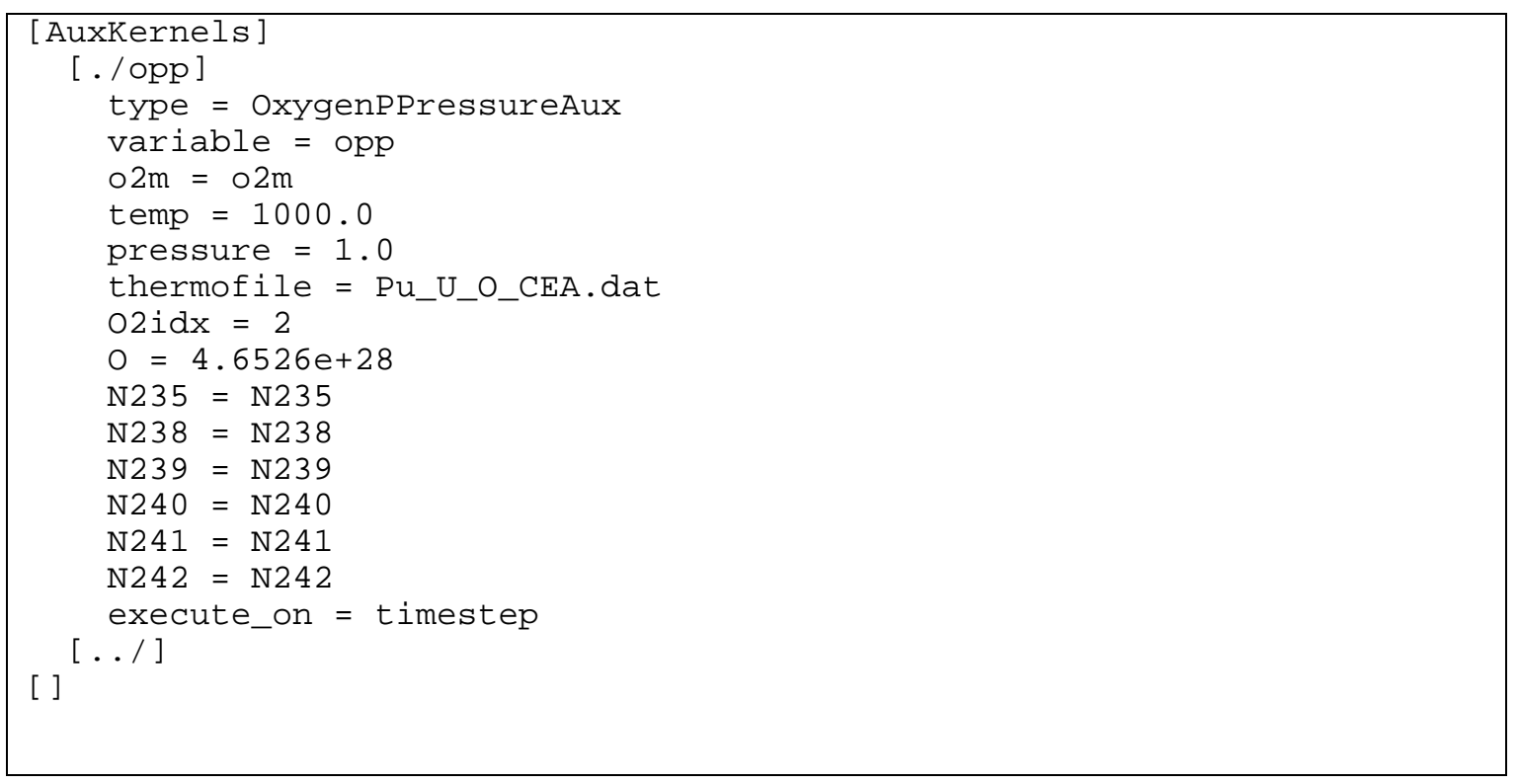

Listing 6. Example input for OxygenPressureAux aux kernel.

In the input block of Listing 6, constant temperature is used although the kernel is capable of coupling with the temperature field. The pressure is provided as a scalar parameter although it can be easily extended in the MOOSE framework to accept the variable values. Parameters N235 - N242 denote coupled variables from the Burnup block. Parameter 0 denotes the number of oxygen atoms in the unit volume calculated from the initial composition of the fuel. Other parameters have the same meaning as in the block described above. The kernel calculates oxygen partial pressure as the main variable and oxygen to metal ratio in the fluorite fuel phase as the secondary coupled variable. The kernel evaluation is fairly straightforward and the main steps of the evaluation are given in Listing 7. 


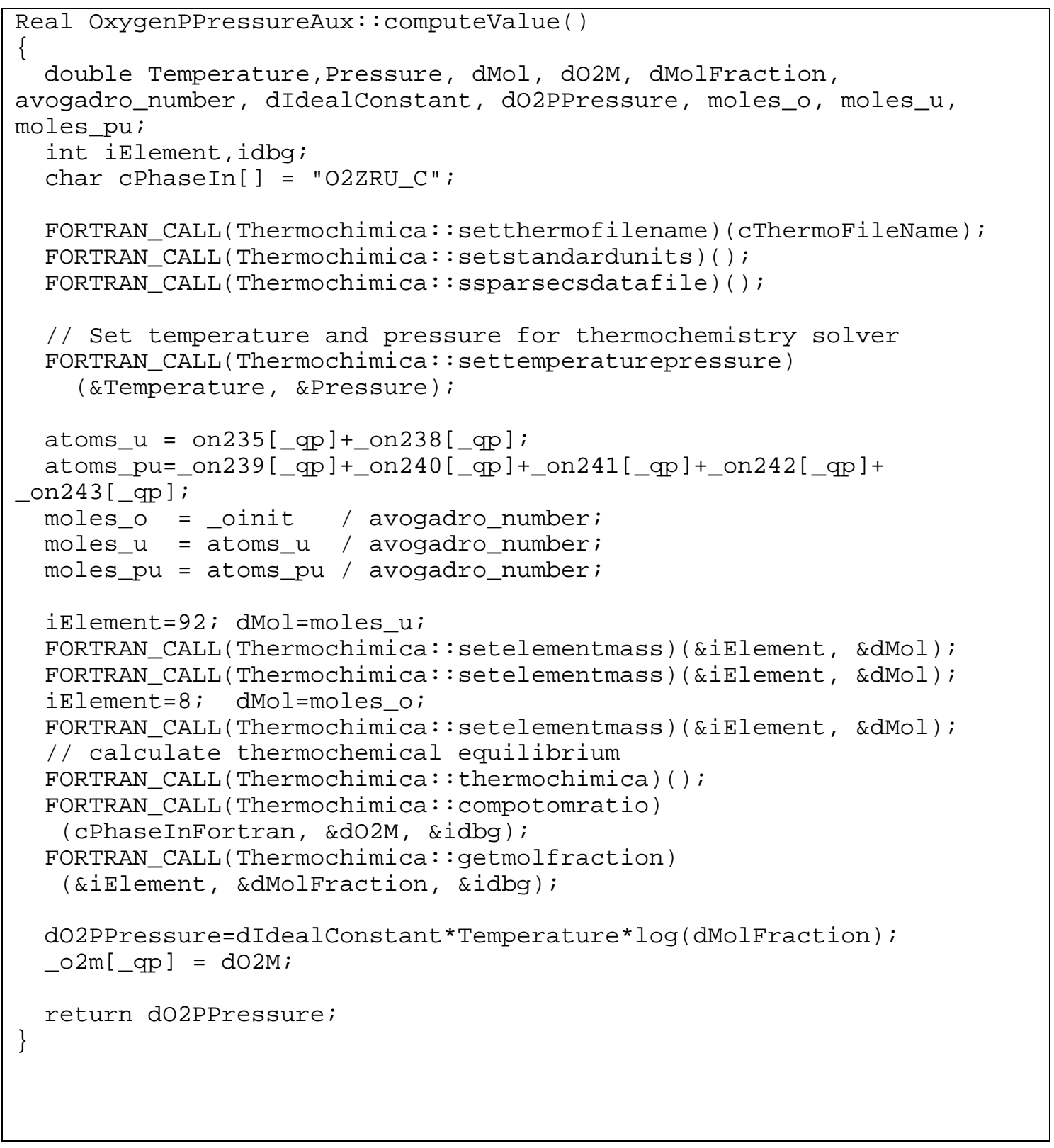

Listing 7. Aux kernel 0xygenPPressureAux evaluation procedure.

As before the main function calls compotomratio and getmolfraction are used to calculate oxygen to metal ratio and mole fraction of $\mathrm{O}_{2}$ in the computed species.

\section{Oxidation Calculations in BISON: Test and Example Problems}

The accuracy of the developed functions and subroutines were validated using simple test and example problems available in BISON. We first show an oxidation state calculation on a simple cubic domain with dimensions given in Figure 8a.. Thermal boundary conditions were applied that impose a temperature gradient on the thermal solution in the domain between $1473 \mathrm{~K}$ and $873 \mathrm{~K}$ as shown in Figure 8b. 


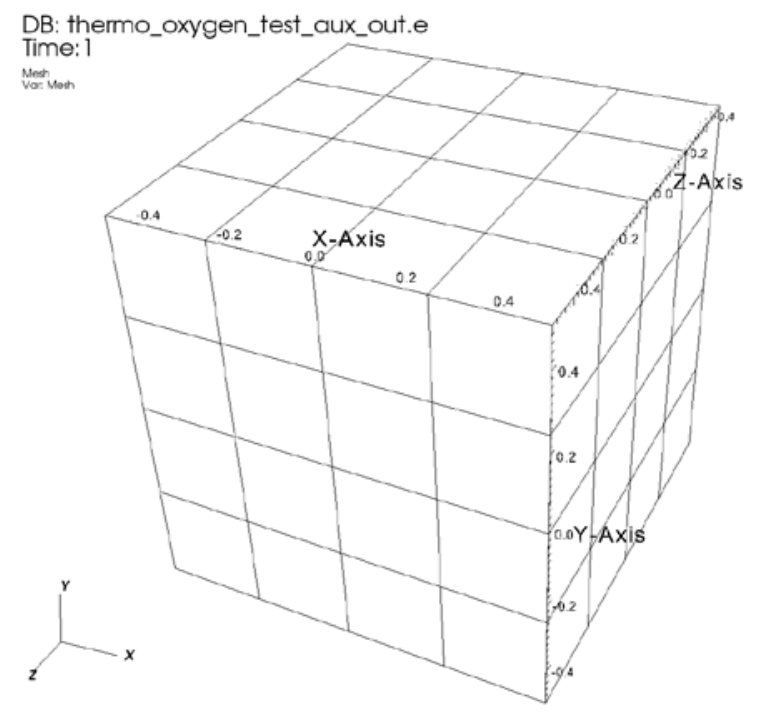

(a)

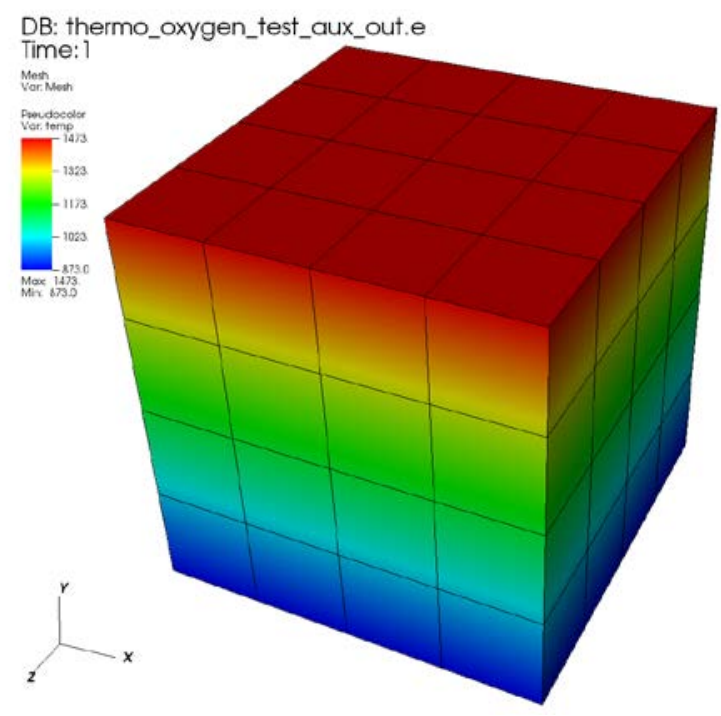

(b)

Figure 8. (a) Problem geometry, and (b) Temperature [K] distribution for the thermal solution.

The elemental composition that was used to initiate the composition of the cubic domain corresponds to the calculated fission product inventory (Figure 5) for the outermost pellet ring in Figure 3 , with the rings centerline at $r / r_{0}=0.9$. By imposing the temperature gradient between the bottom $(873 \mathrm{~K})$ and the top $(1473 \mathrm{~K})$ surface, the values of the oxygen potential across the vertical direction should recover values for at $r / r_{0}=0.9$ curve in Figure 6 . The oxygen potential and oxygen to metal ratio in the fluorite phase computed in BISON by the 0xygenThermochimicaAux auxiliary kernel are shown in Figures 9a and 9b, respectively.

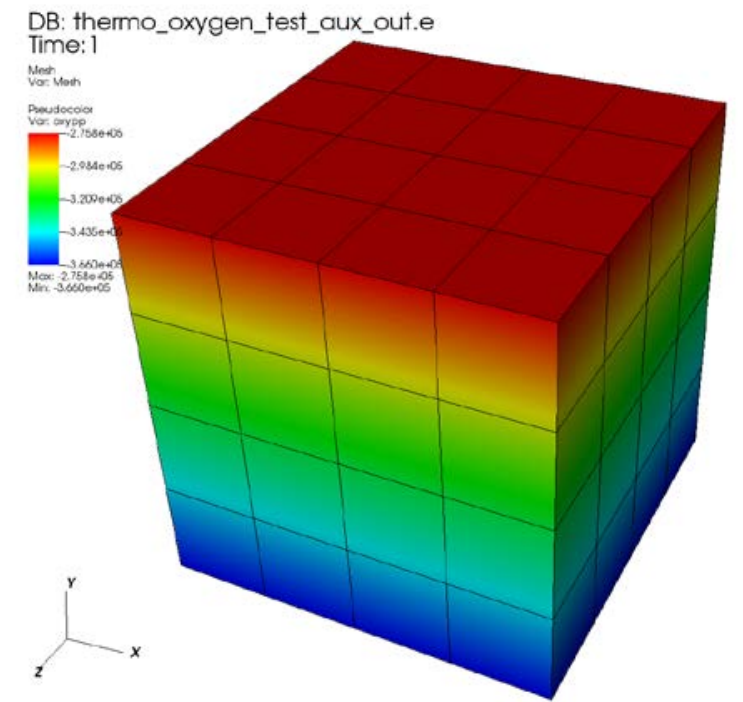

(a)

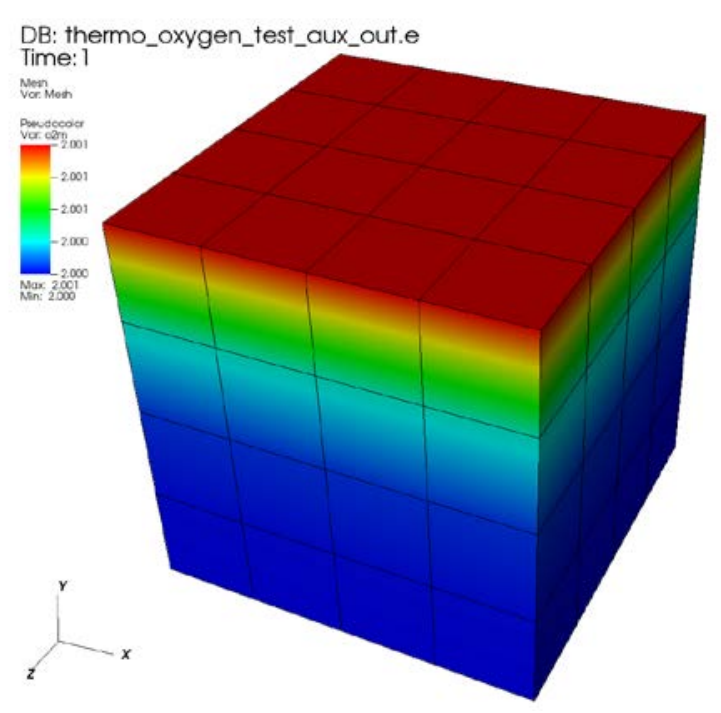

(b)

Figure 9. (a) Oxygen potential [J/mol] and (b) oxygen-to-metal ratio distribution. 
The two extreme values of the oxygen potential in Figure 9a match the values for the curve $r / r_{0}=0.9$ in Figure 6 for temperatures of 600 C (873 K) and 1200 C (1473 K), respectively, and thereby validate the BISON implementation of the oxidation state kernel.

In the next two simulations, a simplified version of the thermodynamics model and the auxiliary kernel 0xygenPPressureAux were used and coupled with the Burnup model in BISON. The existing problems in bison/examples/2D_plane_strain_rod were modified to include the new oxidation state kernel. Figures 10 and 11 show the temperature and oxygen potential distributions for 600s and 133100s. With increasing burnup the oxidation state of the fuel increases as expected due to the limited fission product inventory used in the model.

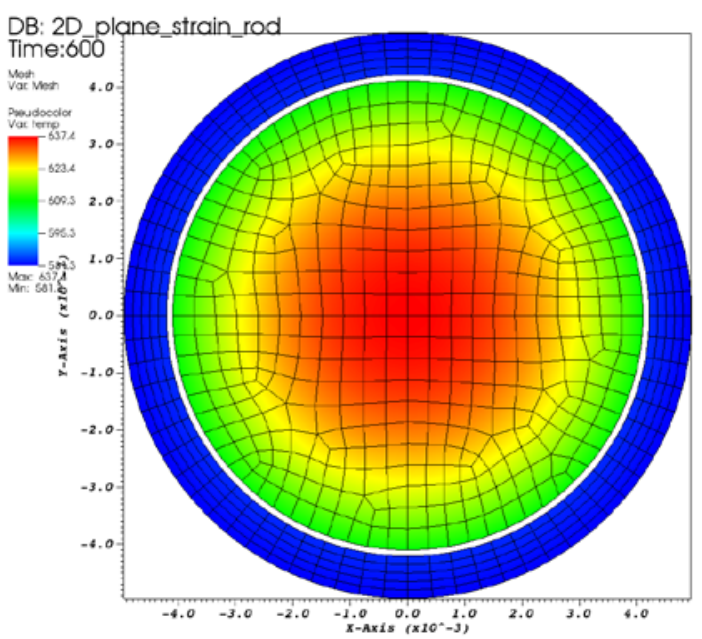

(a)

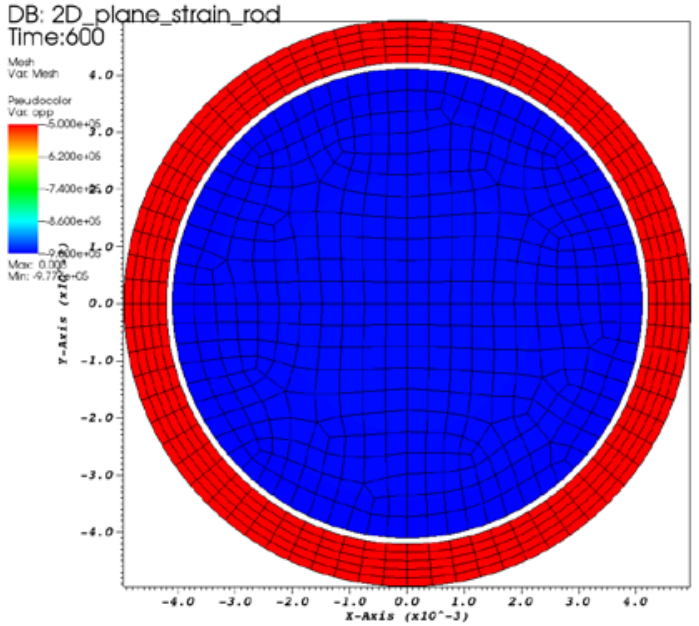

(b)

Figure 10. (a) Temperature $[\mathrm{K}]$ and (b) oxygen potential $[\mathrm{J} / \mathrm{mol}]$ for time $600 \mathrm{~s}$.

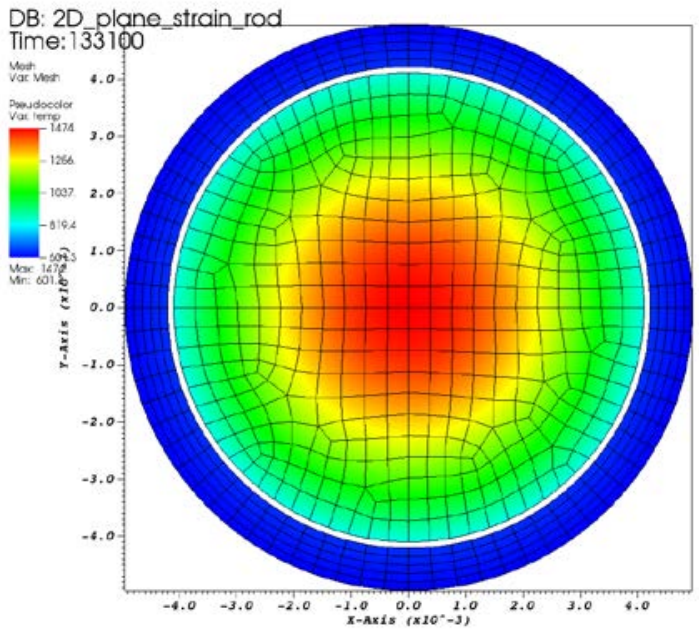

(a)

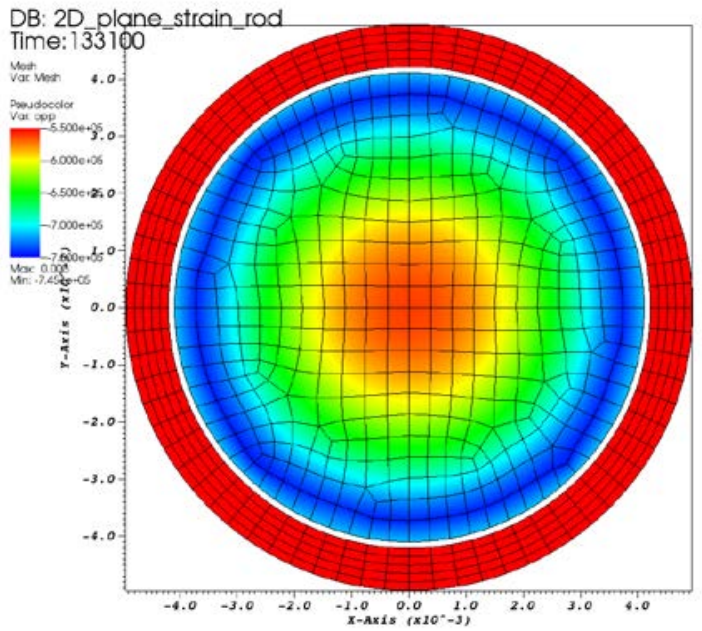

(b)

Figure 11. (a) Temperature [K], and (b) oxygen potential [J/mol] for time $133100 \mathrm{~s}$.

The three-dimensional model in BISON directory bison/examples/3D_rodlet_3pellets was also modified to use the oxidation state kernel. The geometry of the problem is shown in Figure 12. 


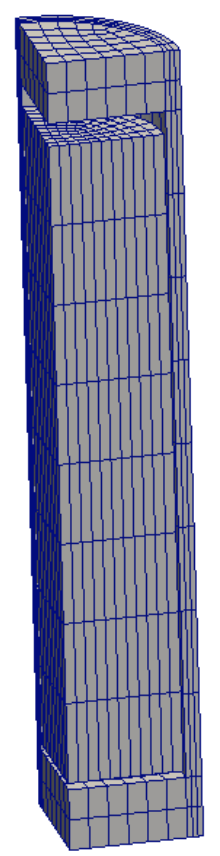

Figure 12. Geometry configuration of the 3D, three pellet model.

Similarly to the previous problem, the model showed increasing oxidation state of the fuel as shown in the Figures 13 and 14.

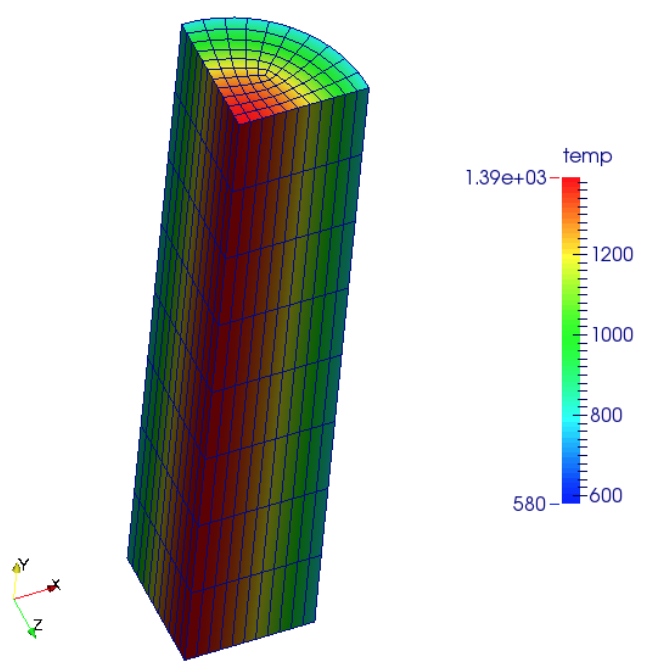

(a)

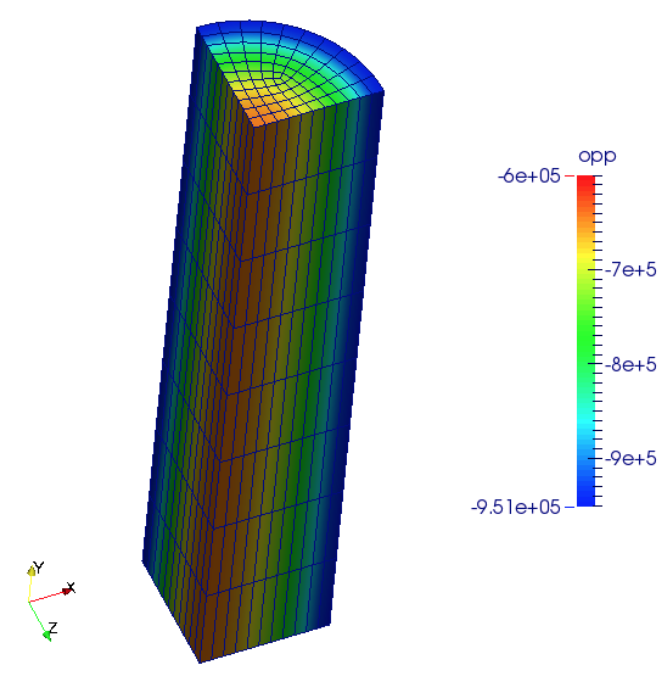

(b)

Figure 13. (a) Temperature [K], and (b) oxygen potential [J/mol] for time $18800 \mathrm{~s}$. 


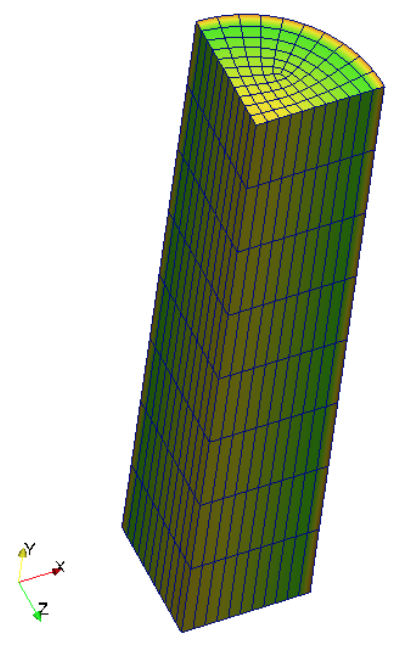

(a)

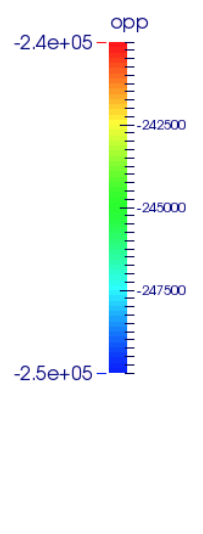

Figure 14. (a) Oxygen potential [J/mol] and (b) oxygen to metal ratio at $1.32828 \mathrm{e}+07 \mathrm{~s}$.

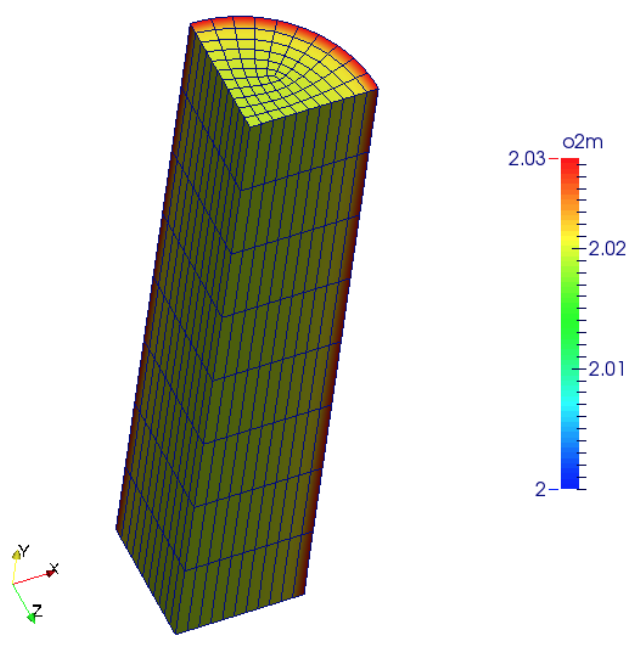

(b)

The above examples were simulated only to illustrate possible coupling in the existing framework and were not intended to investigate physical behavior of the fuel. In these simulations, the burnup process is very oxidizing and the oxygen to metal ratio is relatively high as expected because only uranium and plutonium isotopes are tracked. In order to enable a better estimate of the oxygen potential and the chemical state of the fuel it would be necessary to consider a larger number of fission products and more a comprehensive thermodynamic model.

The oxidation state kernel was also tested for BISON assessment problems in the bison/assessment directory where it performed similarly to the other example problems in the directory bison/examples. The IFA 515.10 Rod A1 fuel rod assessment problem was run with the oxygen potential kernel. The resulting temperature and oxygen potential distribution at the end of the simulation are shown in Figure 15. The radial direction was scaled by factor 20. The clad and expansion thermometer in the center were blacked out in Figure 14b as the value of the oxygen potential variable is zero in those regions. 


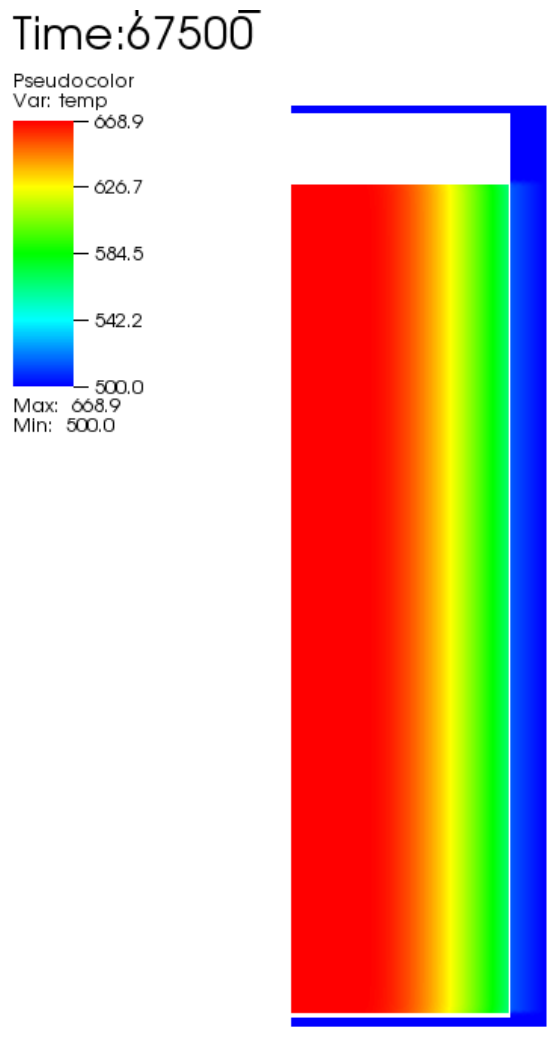

(a)

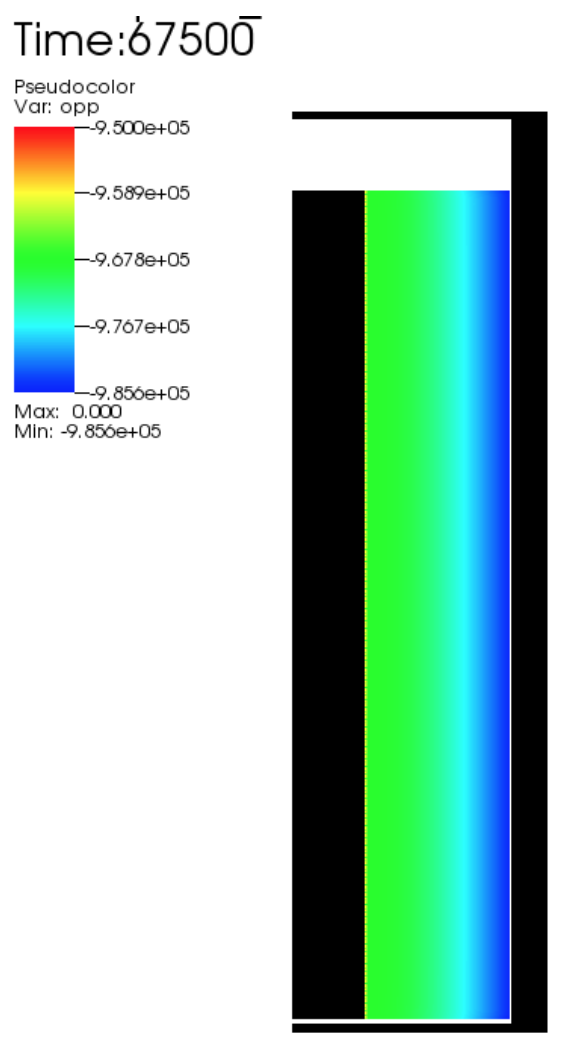

(b)

Figure 14. (a) Temperature [K] and (b) Oxygen potential [J/mol] for IFA 515.10 Rod A1 assessment problem

\section{Summary}

A thermochemical database for LWR fuel containing transuranics and fission products has been developed. The database was used in thermochemical calculations for simulating the composition of high burnup LWR nuclear fuel. The fuel element composition was provided by SCALE computations that used fuel composition and operating conditions from experimental measurements. The thermodynamics models and oxidation state computation modules implementation in BISON were used to simulate oxygen partial pressure values in the fuel and were compared to experiments, which showed good agreement. The developed modules were also demonstrated on small test problems and standard examples in BISON. The modules were coupled with the BISON burnup model which is restricted to tracking only isotopes of uranium and plutonium. The results from these simulations are consistent with the expected results and trends that are in such systems. The developed THERMOCHIMICA modules constitute a unique capability for modeling composition and spatial variation of compounds and phases in nuclear fuel during irradiation, and provide the framework for more accurate modeling of diffusion controlled processes in practical multicomponent systems. 


\section{Acknowledgments}

The authors would like to thank Markus H.A. Piro of Chalk River Laboratory, Chalk River, Canada, for his technical assistance and continuing development of THERMOCHIMICA. The authors would like to thank James E. Banfield of GE Hitachi Nuclear Energy and Kevin T. Clarno of Oak Ridge National Laboratory for developing models for SCALE and running the simulations. Research supported by the U.S. Department of Energy, Office of Nuclear Energy, Nuclear Energy Advanced Modeling and Simulation Program.

\section{References}

[1] M. H. A. Piro, J. Banfield, K. T. Clarno, S. Simunovic, T. M. Besmann, B. J. Lewis, et al., "Coupled thermochemical, isotopic evolution and heat transfer simulations in highly irradiated UO2 nuclear fuel," Journal of Nuclear Materials, vol. 441, pp. 240-251, Oct 2013.

[2] D. Staicu, "Thermal Properties of Irradiated UO2 and MOX," in Comprehensive Nuclear Materials. vol. 2, R. J. M. Konings, Ed., "'" ed: Elsevier, 2012, pp. 439 - 464.

[3] B. J. Lewis, W. T. Thompson, and F. C. Iglesias, "Fission Product Chemistry in Oxide Fuels," in Comprehensive Nuclear Materials. vol. 2, R. J. M. Konings, Ed., "' ed: Elsevier, 2012, pp. 515 - 546.

[4] M. H. A. Piro, S. Simunovic, T. M. Besmann, B. J. Lewis, and W. T. Thompson, "The thermochemistry library Thermochimica," Computational Materials Science, vol. 67, pp. 266-272, Feb 2013.

[5] M. H. A. Piro, T. M. Besmann, S. Simunovic, B. J. Lewis, and W. T. Thompson, "Numerical verification of equilibrium thermodynamic computations in nuclear fuel performance codes," Journal of Nuclear Materials, vol. 414, pp. 399-407, Jul 2011.

[6] M. H. A. Piro and S. Simunovic, "Performance enhancing algorithms for computing thermodynamic equilibria," Calphad-Computer Coupling of Phase Diagrams and Thermochemistry, vol. 39, pp. 104-110, Dec 2012.

[7] D. Gaston, C. Newman, G. Hansen, and D. Lebrun-Grandie, "MOOSE: A parallel computational framework for coupled systems of nonlinear equations," Nuclear Engineering and Design, vol. 239, pp. 1768-1778, Oct 2009.

[8] J. D. Hales, S. R. Novascone, R. L. Williamson, D. R. Gaston, and M. R. Tonks, "Solving Nonlinear Solid Mechanics Problems with the Jacobian-Free Newton Krylov Method," Cmes-Computer Modeling in Engineering \& Sciences, vol. 84, pp. 123-153, Mar 2012.

[9] M. H. A. Piro, S. Simunovic, and T. M. Besmann, "Thermochimica User Manual v1.0," Oak Ridge National Laboratory, Oak Ridge2012.

[10] C. T. Walker, V. V. Rondinella, D. Papaioannou, S. Van Winckel, W. Goll, and R. Manzel, "On the oxidation state Of UO2 nuclear fuel at a burn-up of around $100 \mathrm{MWd} / \mathrm{kgHM}$," Journal of Nuclear Materials, vol. 345, pp. 192-205, Oct 2005.

[11] I. Gauld, O. Hermann, and R. Westfall, "ORIGEN Scale System Module to Calculate Fuel Depletion, Actinide Transmutation, Fission Product Buildup and Decay, and Associated Radiation Terms," 2005.

[12] K. Lassmann, C. Ocarroll, J. Vandelaar, and C. T. Walker, "The radial-distribution of plutonium in high burnup UO2 fuels," Journal of Nuclear Materials, vol. 208, pp. 223-231, Feb 1994.

[13] C. Gueneau, N. Dupin, B. Sundman, C. Martial, J. C. Dumas, S. Gosse, et al., "Thermodynamic modelling of advanced oxide and carbide nuclear fuels: Description of the U-Pu-O-C systems," Journal of Nuclear Materials, vol. 419, pp. 145-167, Dec 2011. 
[14] H. Kleykamp, "Chemical-State of LWR High Power Rods Under Irradiation," Journal of Nuclear Materials, vol. 84, pp. 109-117, 1979.

[15] M. H. Kaye, B. J. Lewis, and W. T. Thompson, "Thermodynamic treatment of noble metal fission products in nuclear fuel," Journal of Nuclear Materials, vol. 366, pp. 8-27, Jun 2007.

[16] C. Bale, P. Chartrand, S. A. Degterov, G. Eriksson, K. Hack, R. Ben Mahfoud, et al., "FactSage thermochemical software and databases," Calphad-Computer Coupling of Phase Diagrams and Thermochemistry, vol. 26, pp. 189-228, Jun 2002.

[17] M. G. Adamson, E. A. Aitken, S. K. Evans, and J. H. Davies, "Oxygen Redistribution and its Measurement in Irradiated Oxide Fuels," presented at the Thermodynamics of Nuclear Materials 1974, Vienna, Austria, 1975.

[18] K. Une, Y. Tominaga, and S. Kashibe, "Oxygen Potentials and Lattice-Parameter of Irradiated BWR Fuels," Journal of Nuclear Science and Technology, vol. 28, pp. 409-417, May 1991.

[19] H. Matzke, "Oxygen Potential Measurements in High Burnup LWR UO2 Fuel," Journal of Nuclear Materials, vol. 223, pp. 1-5, May 1995. 